\title{
An Analysis of the Overhead and Energy Consumption in Flooding, Random Walk and Gossip based Resource Discovery Protocols in MP2P Networks
}

\author{
Ajay Arunachalam, Ohm Sornil \\ Department of Computer Science, \\ National Institute of Development Administration (NIDA), Thailand \\ ajay.abhi@grads.nida.ac.th,osornil@as.nida.ac.th
}

\begin{abstract}
All major mobile communication architectures are mainly centralized. When the mobile devices are switched on it will search for nearby base station or access point. The content being searched is mostly stored in a centralized directory manner. Peer-to-Peer platform can be one of the best possibilities to overcome the restrictions and resolve issues incurred due to centralization. Mobile environment poses additional challenges on such P2P networks - due to limited resources, dynamic and wireless network characteristics, heterogeneity of nodes, limitations on processing power and wireless bandwidth. Hence resource discovery becomes further challenging. Even today mostly the traditional methods like flooding, random walk or gossip based forwarding methods have to be considered along with major limitations and drawbacks. Further in Mobile Peerto-Peer (MP2P) system the energy aspect is very crucial with regards to the participation of nodes in the system. The search failure rate may increase if a mobile device uses all its energy and hence not participate in the resource discovery process. In this paper, we simulate the existing standard flooding, random walk and gossip based resource discovery algorithms on a P2P Mobile Adhoc Network (MANET) and studied their performance under such highly dynamic mobile network scenario. The efficiency of the resource discovery protocols are validated through extensive NS-2 simulations.
\end{abstract}

Keywords-MANET; P2P; Analysis; Search; MP2P; Peer-to-Peer; Survey

\section{INTRODUCTION}

\section{Peer-to-peer (P2P) Networks}

In Peer-to-Peer networks the nodes so called "peers" share computer resources and services by point to point exchange between them. These resources or services may include information exchange, file sharing, disk storage, cache storage and power cycles. Also mostly abbreviated as P2P where every peer takes advantage of existing computing power, computer storage and networking connectivity among each other. All nodes have equal roles which can act as clients and servers both in a fully distributed P2P system. P2P systems are mainly classified as centralized \& decentralized. The decentralized P2P systems further can be structured or unstructured. In centralized P2P architecture such as Napster [1], central index servers are maintained which can cause bottleneck. Whereas the decentralized architecture eliminate the dependency of central servers allowing point-to-point service. In decentralized structured P2P system such as CAN [2], Chord [3] , Pastry [4] the arrangement of the peers are structured \& well-organized on the basis of distributed hash functions. These structured P2P system may increase the complexity wherein the nodes are in continuous movement. While in the decentralized unstructured model there is neither any predefined rule nor central index on the arrangement of peers or placement of the file. The most famous example being Gnutella [5]. Even today P2P have to be focused on further, especially due to large amount of traffic it generates thereby causing overhead. In [6] authors discusses various problems of wireless \& mobile limitations in $\mathrm{P} 2 \mathrm{P}$ and suggests how to use current trends in technology to help boost peer-topeer architecture in mobile setup. With development of Mobile P2P application the use of mobile nodes itself can be useful to build a P2P network. Their work describes the use of a Mobile P2P middleware framework. They present an architecture based on JXTA middleware for using the special features of mobile nodes such as mobility \& node capability to the maximum.

\section{P2P MANET}

A Mobile Adhoc Network (MANET) is a kind of wireless adhoc network that consists of mobile devices connected without wires. Incorporating peer-to-peer network characteristics in mobile ad-hoc networks can be called as P2P MANET or Mobile P2P (MP2P) network. Mobile peer-to-peer systems are systems that can either connect together instantly in an ad-hoc fashion or use telecom operator's mobile networks to connect to P2P system on which they may collaborate with fixed peers [7]. Peer-to-Peer networks have shown their potential advantage over centralized approach. Inspired by the fixed domain, peer-to-peer networks are now penetrating the wireless and mobile domain. The first mobile peer-to-peer service was a dating client [8]. The main idea was that people with the same interest in meeting other people could specify their interests and needs by using a mobile application. Recently the trend is shifting towards social mobile networking and cooperative networking which are being implemented in this field. The MP2P encompasses different architectures such as point to point, meshed networks and cooperative networks. But a simple combination of P2P and MANETs also poses great challenges because P2P operates on the application layer while MANET operates on the network layer. Therefore, just simply adopting P2P overlay protocol over MANET is undesirable due to the limitations of infrastructure, mobility, power consumption issues etc. Further the limited feature of adhoc network decreases the query success rate and connectivity in P2P 
system since nodes may be moving to different locations. The dynamic topology of such adhoc network rules out the possibility of deploying services based on dedicated single servers. So a decentralized approach like P2P is a suitable solution to be adopted in such environments. Over past years, P2P techniques have their applicability to MANETs, VANETs, etc. A brief survey of P2P solutions \& their applications under MANET is discussed [9]. They list out the various P2P related work done for MANETs. Use of P2P topology in MANET can be efficient and useful for various services involving mobile nodes in such system.

\section{Background and Related Work}

Peer-to-Peer technology is widely used in wired infrastructure or internet domain that has wired layout. When comparing to wired P2P networks, adhoc infrastructure less network require improved and adaptable resource discovery strategies, due to difference between the mobile and internet transmission environment. Resource discovery protocol is one of the important demanding areas of P2P technology and becomes more challenging task in such Mobile scenario [10]. The resource discovery mechanism over P2P networks depend on structure classified into two broad types - unstructured and structured. The searching mechanism in P2P is classified broadly as centralized index based, flooded request model and structured lookup model. With development of wireless network over past years and wide acceptance of peer-to-peer networks the MP2P [11] has emerged. Compared to traditional $\mathrm{P} 2 \mathrm{P}, \mathrm{MP} 2 \mathrm{P}$ has many limitations [12] which make resource search \& information sharing inefficient in mobile network scenario. [13] provides the limitation of application of peer-topeer services in multi-hop networks such as MANETs. Further they provide a survey of various research carried out in this area. Direct application of P2P over MANET provides a low performance. So potentially various alternatives are to be considered such as focusing on Overlay P2P protocol, Overlay adaptation, integrated approach, efficient routing algorithm, location based overlay adaptation, overlay peer management etc. One of the most important aspects of MP2P is efficient resource search and routing. New resource searching algorithms based on characteristics of Mobile P2P networks [14] have to be focused on. The resource discovery mechanisms in MANETs are related to routing protocols which are further classified as proactive $\&$ reactive. Proactive routing protocols build and maintain routing tables periodically whereas the nodes in reactive routing protocols broadcast the query to the whole network and determine routes on-demand as and when needed. Further with its versatile changing environment and along with its limited battery \& memory constraints, effective use of limited energy of mobile devices is very critical. Especially in Mobile Peer-to-Peer (MP2P) system if a mobile device uses all its energy, then it can no longer participate in the system. Mobile environment being dynamic in nature has to utilize the battery power efficiently. The routing for Mobile P2P environment is very challenging and lot of research effort is done in this area. In [15] authors proposed a routing protocol limiting the energy consumption. They came up with the node replacement scheme and route changing scheme for double layered mobile
P2P system. A different search approach was suggested by [16] where they proposed a self-aware scheme that form groups based on the common interest and then accordingly each node maintain a list of familiar nodes \& neighbor nodes based on the positive reply send by the node. Both [15][16] are based on Super node. The ORION [17] system was developed as P2P file sharing system for Mobile Adhoc Networks. Gossip protocols can also be used for resource search in such dynamic self-organizing networks. A brief review of gossip protocol with its pros and cons are discussed in [18]. The major disadvantages of gossip protocols are the message duplication, high latency of message delivery, slow speed and unstability.

The above section of paper discussed about MANET and the problems in implementing P2P network on MANET. It also discusses the various ways of how resource discovery has been carried out in past in such network. The rest of the paper is organized as follows: Section II is the algorithm description of the existing resource discovery protocols. Section III discusses about the simulation environment and the parameters used for measuring the performance metrics. Section IV analyzes the experimental results. Finally, section $\mathrm{V}$ concludes with a summary and some directions for future work.

\section{P2P RESOURCE DISCOVERY ALGORITHMS UNDER MANET}

For understanding the algorithms in a simple manner, let us consider the following simple network scenario.

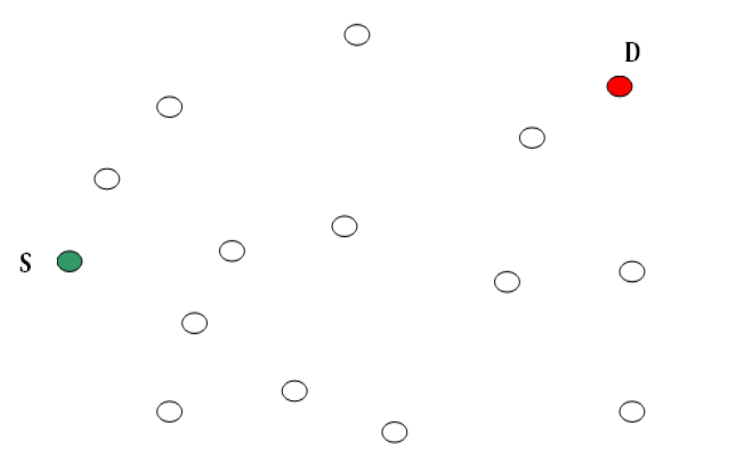

Figure 1. The Example P2P Network

- The green node ' $\mathrm{S}$ ' initializes the resource query

- And red node 'D' actually has that resource.

(Here in this example, we only consider one querying node and one resource containing node for the simplicity of understanding the algorithms - but the implementation will be much more complex than this simple scenario) 
In our simulation and the algorithms, the meaning of resource discovery in a standard P2P system is nothing but discovering the address of the node in the network which actually has that resource. So, here in our implementation, the reply will be send by a resource containing node, and the requesting node will resolve the address of the resource provider.

\section{A. The Standard Flooding Based Resource Discovery Method.}

The following steps outline the standard flooding based resource discovery method which is to be implemented under MANET.

1. The Resource Request is starting from Node ' $\mathrm{S}$ '

2. First the Node ' $\mathrm{S}$ ' will send a 1-hop broadcast with message "S Need Resource X"

3. All the 1-hop neighbors receiving that message will store the sequence ID of the message in \{processed messages list $\}$ so that it will not forward the same message again in case it will be received over another path.

4. If a neighbor is the owner of the Resource ' $\mathrm{X}$ ' (the node ' $\mathrm{D}$ ' in this case), then it will send an exclusive multi-hop reply to the Source ' $S$ ' (based on the path resolved by routing protocol). But even after the node ' $D$ ' sending the resource reply, all other nodes which receive the request may meaninglessly resume forwarding the message until reaching all the nodes in the network.

5. Otherwise after that all the 1-hop neighbors receiving that message will continue forwarding the message until reaching the node ' $\mathrm{D}$ ' (since node $\mathrm{D}$ already processed that request, it will silently drop the duplicate requests that received over other paths).

Here the message is flooded to all the nodes in the network.

\section{B. The Standard Random Walk Based Resource Discovery Method}

The following steps outline the standard random walk based resource discovery method which is to be implemented under MANET.

1. The Resource Request is starting from Node ' $\mathrm{S}$ '. (here the current node $\mathrm{C}=\mathrm{S}$ ) - The request will contain a message like "S Need Resource X"

2. Get Neighbor list $\{\mathrm{N}\}$ of the current node ' $\mathrm{C}$ ' from the routing layer neighbor list information.

3. Randomly select one neighbor ' $n$ ' from the list $\{\mathrm{N}\}$

4. The request is then passed to only one randomly selected neighbor node ' $n$ ' over unicast transmission. (here even though it is a 1-hop communication, the unicast transmission mechanism of MANET will find the next hop information of ' $n$ ' from the routing table and forward the packet to that hop)

5. On receiving the message, if that randomly selected neighbor node ' $n$ ' is the owner of the Resource ' $X$ ' (the node ' $D$ ' in this case), then it will send a exclusive multihop reply to the Source ' $\mathrm{S}$ ' (based on the path resolved by routing protocol) and the query get terminated at that point and the process will get terminated.

6. Otherwise, the neighbor node ' $n$ ' becomes the current node ' $\mathrm{C}$ ' and the same process is repeated from step 2 and this continues until "randomly finding" the destination ' $\mathrm{D}$ ' which has the resource.

Here the message is not flooded to all the nodes in the network but the probability of reaching ' $\mathrm{D}$ ' is low (because a request packet may be lost in between the process) and the random process may take much time to find that resource. But overhead will be expected to be low since the message is not broadcasted to all the neighbors and the message is only moved to one node at a time.

\section{The Standard Gossip Based Resource Discovery Method}

The following steps outline the standard gossip based resource discovery method which is to be implemented under MANET.

1. The Resource Request is starting from Node ' $S$ ' (here the current node $\mathrm{C}=\mathrm{S}$ ) - The request will contain a message like "S Need Resource X"

2. Get Neighbor list $\{\mathrm{N}\}$ of the current node ' $\mathrm{C}$ ' from the routing layer neighbor list information.

3. Randomly select ' $g$ ' number of neighbors from the list $\{\mathrm{N}\}$ and forward the gossip message to the first node of the selected neighbors ' $\mathrm{g}$ '.

4. Schedule the gossip message for the remaining selected neighbors in ' $\mathrm{g}$ ' in an interval I. (It means, a node may gossip with more than one node in a periodic manner) (in step 3 and 4 the request is then gossiped to ' $\mathrm{g}$ ' randomly selected neighbor nodes over unicast transmission. (here even though it is a 1hop communication, the unicast transmission mechanism of MANET will find the next hop information from the routing table and forward the packet to that hop)

5. On receiving the message, if any one of those randomly gossiped neighbor node is the owner of the Resource ' $X$ ' (the node ' $D$ ' in this case), then it will send a exclusive multihop reply to the Source ' $S$ ' (based on the path resolved by routing protocol) and the query get terminated at that point(since the address ' $S$ ' will be available in the received message itself) and the process will get terminated at that node 
but without knowing that reply, the remaining randomly gossiped nodes resume the gossip even if any other node already sent reply for that request.

6. Each gossiped neighbors ' $\mathrm{g}$ ' becomes the current node ' $\mathrm{C}$ ' and the same process is repeated from step 2 and this continues until "randomly finding" the destination ' $\mathrm{D}$ ' which has the resource. (So the gossip will spread in more than one direction at a time)

Gossip Protocol involves periodic message exchange between pair of nodes.

The standard way of implementing random walk and gossip for P2P resource discovery under MANET has some issues.

Both in random walk and gossip, the message is passed to a randomly selected neighbor node using unicast message transmission. But such unicast transmissions will depend on the routing layer information. But, in MANET, the nodes may be in continuous movement. So, the neighbors of all the nodes will rapidly change over time. The unicast message transmission may fail very often due to the rapid change in network topology. And this will initiate frequent re-route discovery and increase the routing overhead. So even for finding the route of nearest neighbors the random walk and gossip based method will try to discover route at each and every hop(while passing message to another node) and mobility \& link failures induces frequent re-route discovery thereby increasing the overhead.

\section{SIMULATION ENVIRONMENT}

We have implemented all the resource discovery algorithms on ns-2 [19] network simulator. We used network simulator version ns-2.35 for all our experiments. We have simulated a typical P2P network resource discovery scenario as follows:

We setup some portion of the nodes as resource providing nodes and some portion of the nodes as resource requesting nodes. And generated few random resource discovery requests from them at uniform intervals. We used AODV [20] as the routing protocol. The neighbor information and the neighbor count for each node are resolved from the routing layer information.

\section{A. The Parameters of the Simulation}

\section{Common Parameters}

Channel WirelessChannel

$\begin{array}{ll}\text { Propagation Model } & \text { TwoRayGround } \\ \text { Physical Model } & \text { WirelessPhy } \\ \text { MacProtocol } & 802 \_11 \\ \text { QueueType } & \text { DropTail PriQueue } \\ \text { Queue Length } & 50 \\ \text { Antenna Type } & \text { OmniAntenna } \\ \text { Energy Model } & \text { Battery } \\ \text { Initial Node Energy } & \text { 500 Joules } \\ \text { Routing Protocol } & \text { AODV } \\ \text { P2P RD Protocol } & \text { StdFlooding, StdRandomWalk, } \\ & \text { StdGossip }\end{array}$

The Nodes and the Network Scenario

$\begin{array}{ll}\text { Number of Mobile Nodes } & 70,80,90 \\ \text { Resource Providing Nodes } & 10 \\ \text { Topography Size } & 1000 \mathrm{~m} \times 1000 \mathrm{~m} \\ \text { Pause Time } & 20.00 \mathrm{sec} \\ \text { Max speed } & 20.00 \mathrm{~m} / \mathrm{s}\end{array}$

P2P Application Related Parameters

$\begin{array}{lc}\text { P2PApplicationPort } & 6346(\text { Gnutella) } \\ \text { OneHopBroadcastAddress } & -1 \\ \text { P2PResourceRequestMessageSize } & 100 \mathrm{bytes} \\ \text { P2PresourceReplyMessageSize } & 100 \mathrm{bytes} \\ \text { BroadcastDelay } & 0.01 \mathrm{sec} \\ \text { TransmissionProbability } & 100(=1) \\ \text { GossipInterval } & 1 \mathrm{sec}\end{array}$

(If a node receives a Gossip, after sharing it immediately with a node after GossipDelay seconds, the GossipInterval is the time it should wait for sharing it with another node)

NoGossipsPerRequestPerNode 2

GossipDelay $\quad 0.01$

(If a node receives a Gossip, then GossipDelay is how much time it should wait for sharing it with first random node)

\section{B. Evaluating the Metrics}

Generally, there are many metrics for evaluating the performance of an algorithm under MANET. Since a typical 
P2P network resource discovery scenario is peculiar in nature, we need to modify the most of the standard metrics. As of now we focus on two of the important metrics to measure the performance of the $\mathrm{P} 2 \mathrm{P}$ resource discovery algorithms under MANET. They are:

- P2P Network Overhead

- Avg. Consumed Energy of P2P Network

\section{A) P2P Network Overhead}

In this work we measure the overhead in terms of the total number of generated and forwarded routing message at the Network Layer.

\section{B) Avg Consumed Energy of P2P Network}

Generally, the Consumed Energy is calculated as the average energy consumed by all the nodes of the network for the entire time of the simulation. It is measured in Joules and can be calculated as follows:

$$
E_{A v g}=\frac{1}{N} \sum_{i=1}^{N} I E_{i}-F E_{i} \text { (in Joules) }
$$

Where,

$\mathrm{E}_{\mathrm{Avg}}$ is the calculated avg. consumed energy of $\mathrm{P} 2 \mathrm{P}$ network in Joules

$\mathrm{N}$ is the total number of nodes in the P2P network

$\mathrm{IE}_{\mathrm{i}}$ is initial battery energy of the node I

$\mathrm{FE}_{\mathrm{i}}$ is final battery energy of the node I

\section{RESULTS AND DISCUSSION}

The following bar chart shows the comparative performance of the traditional algorithms in terms of overhead (measured in terms of generated routing packets). As shown in this graph, the performance of flooding is the best as compared to other methods. The standard random walk and standard gossip algorithms are very poor because, there was much routing overhead in their design due to the unicast nature of message forwarding mechanism.

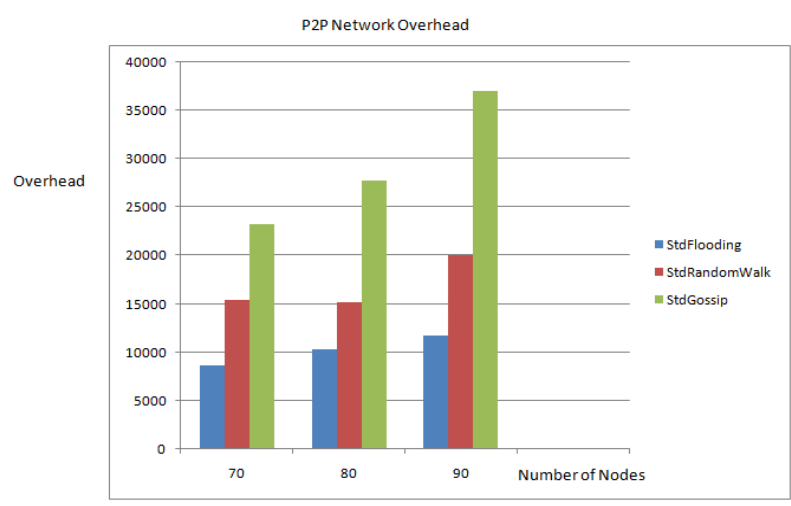

Figure 2. Comparison of Network Overhead
The following bar chart shows the comparative performance of the three resource discovery algorithms in terms of Energy consumption. As shown in this graph, the standard random walk and the standard gossip algorithm consume much battery power because, there was much routing overhead in their standard design due to the unicast nature of message forwarding mechanism which causes frequent re-route discovery as a result of rapid mobility of the nodes. Whereas the flooding technique consumes less energy only because of the use of broadcast in the message forwarding design.

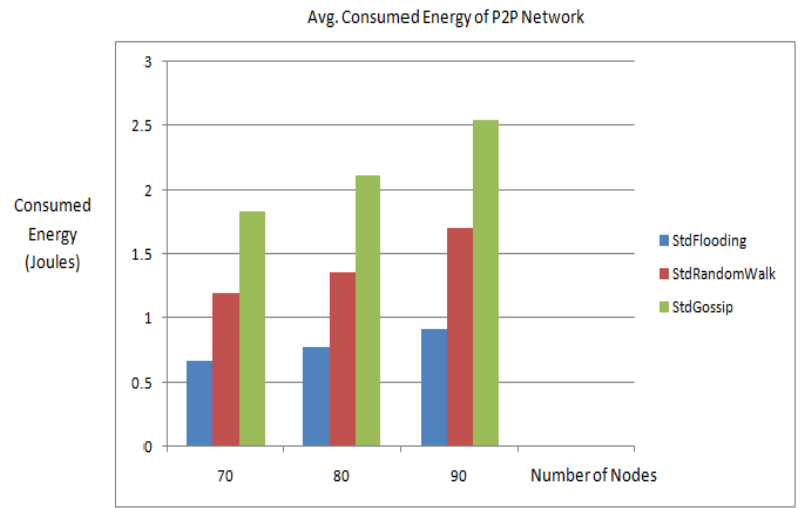

Figure 3. Comparison of Average Consumed Energy

\section{A. Some Interesting Observations and Findings}

- Most of the previous results of P2P implementations are only available for wired like network communication scenario where the random walk and gossip based methods are better than simple flooding. But these methods i.e. the random walk and gossip behaved entirely different under the MANET.

- So, according to our understanding of the results of the existing (P2P resource discovery) systems, and the results of our experiments under MANET, we can say that the randomwalk and gossip based methods may perform better than flooding only under wired context - but certainly not under MANET, as the neighbor discovery and multiple unicast message forwarding in case of both these methods creates lot of routing overhead and it leads to overall poor performance.

- Even up to now the most popular P2P file sharing application "Gnutella" is using a flooding based resource discovery - it may be the practical reason for using the flooded based resource discovery mechanism.

- While using the unicast message transmission in case of the standard random walk, overhead is higher than flooding

- While using unicast message transmission in the case of standard gossip, the overhead is even higher than standard random walk 


\section{CONCLUSION AND FUTURE WORK}

The work describes the issues of implementing random walk and gossip based resource discovery mechanism for Peer-to-Peer network over MANET. We verified the same with repeated simulations for different network scenario and for different number of nodes. We measured the overhead and energy consumption of the nodes involved during the search process. We observed an interesting phenomena where random walk and gossip based techniques performed poor than simple flooding under MANET in terms of network overhead and avg. energy consumed.

Under MANET, if we implement random walk and gossip based methods based on the unicast message transmission - i.e., for sending the packet to the randomly selected neighbor, if we use unicast, then the performance in terms of overhead is poor.

In this paper we have verified two metrics i.e. overhead and battery power consumed. Further we will verify all the other remaining performance metrics to measure the various factors such as Success rate, Response time, MAC Load, Throughput/Bandwidth, Hop to Hop Delay, Packet dropped, Routing Load etc. We will also verify the same by varying the mobility and study their impact on such high density network. Our future work will also focus on addressing these issues observed to reduce the overhead and suggest an alternative way to improve the performance of traditional algorithms to suit such dynamic network.

\section{REFERENCES}

[1] L. J. Hoffman, "Napster and Other Internet Peer-to-Peer Applications," pp. 1-22.

[2] S. Ratnasamy, P. Francis, and M. Handley, A scalable contentaddressable network. 2001.

[3] I. Stoica, R. Morris, and D. Karger, "Chord: A scalable peer-to-peer lookup service for internet applications," ACM SIGCOMM ..., 2001.

[4] A. Rowstron and P. Druschel, "Pastry: Scalable, decentralized object location, and routing for large-scale peer-to-peer systems," Middlew. 2001, no. November 2001, 2001.

[5] Y. Chawathe and S. Ratnasamy, "Making gnutella-like p2p systems scalable," Proc. ..., pp. 407-418, 2003.

[6] R. Motta and J. Pasquale, "Wireless P2P : Problem or Opportunity ?,” no. c, pp. 32-37, 2010

[7] M. Ad and H. Networks, "An Overview of Mobile Ad Hoc Networks : Applications and Challenges," pp. 60-66.

[8] D. Hutchison and S. Fdida, Mobile peer to peer ( $22 p)$ - A tutorial guide. Wiley publication, 2009.

[9] E. Spaho, E. Kulla, F. Xhafa, and L. Barolli, "P2P Solutions to Efficient Mobile Peer Collaboration in MANETs," 2012 Seventh Int. Conf. P2P, Parallel, Grid, Cloud Internet Comput., pp. 379 383, Nov. 2012
[10] D. Qu, X. Wang, and M. Huang, "Research on locality for resource discovery over mobile P2P networks," IET Int. Commun. Conf. Wirel. Mob. Comput. (CCWMC 2009), pp. 425-428, 2009.

[11] V. Kalogeraki, "Mobile peer-to-peer computing: challenges, metrics and applications," Proc. 6th Int. Conf. ..., pp. 331-332, 2005.

[12] T. Repantis and V. Kalogeraki, "Data dissemination in mobile peerto-peer networks," ... 6th Int. Conf. Mob. ..., pp. 211-219, 2005.

[13] M. C. Castro and A. J. K. C. Chiasserini, "Peer-to-Peer Overlay in Mobile Ad-hoc Networks," pp. 1-37.

[14] E. Kang and U. Kim, "Connection-Degree Based Search and Replication in Mobile Unstructured Peer-to-Peer Networks," 2007 Int. Conf. Converg. Inf. Technol. (ICCIT 2007), pp. 919-924, Nov. 2007.

[15] J.-H. Lee, J.-W. Song, Y.-H. Lee, and S.-B. Yang, "An EnergyEffective Routing Protocol for Mobile P2P Systems," 2009 4th Int. Symp. Wirel. Pervasive Comput., pp. 1-5, Feb. 2009.

[16] L. Zhang and J. Liu, "Efficient Search Scheme in Mobile Peer-toPeer Network," 2012 Int. Conf. Ind. Control Electron. Eng., pp. 1235-1238, Aug. 2012

[17] A. Klemm, "A special-purpose peer-to-peer file sharing system for mobile ad hoc networks," ... Conf. 2003. VTC ..., pp. 2758-2763, 2003.

[18] R. Friedman and D. Gavidia, "Gossiping on MANETs: the Beauty and the Beast," ACM SIGOPS ..., no. October, 2007.

[19] "Network Simulator - ns-2." [Online]. Available: http://www.isi.edu/nsnam/ns/.

[20] C. E. Perkins, M. Park, and E. M. Royer, "Ad-hoc On-Demand Distance Vector Routing." 